\title{
Maternal gut microbes shape gut microbiota in zebra finch chicks
}

\author{
Cheng-Yu Chen \\ Chih-Kuan Chen \\ Yi-Ying Chen \\ Andrew Fang \\ Grace Tzun-Wen Shaw \\ Chih-Ming Hung \\ Daryi Wang
}

\section{Video Byte}

Keywords: Microbiome, Avian microbiota, Host-microbiome interactions, Maternal effects, Gut microbiota assembly, Maternal microbial transmission, zebra finch, Taeniopygia guttata, fostering experiments, maternal microbes, but microbiota, passerine, society finch, Lonchura striata domestica, birds

Posted Date: November 12th, 2020

DOI: https://doi.org/10.21203/rs.3.rs-106688/v1

License: (c) (i) This work is licensed under a Creative Commons Attribution 4.0 International License. Read Full License 


\section{Abstract}

Microscopic changes occurring inside newborns may have a huge effect on how they develop. Microbes residing inside the developing newborn - gut microbiota - are influenced by their delivery, diet, environment, and parenting, and the species within a developing newborn can influence host health, fitness, and behavior. However, exactly how microbes are passed to newborn birds remains unclear. A recent study examined this phenomenon in zebra finch chicks. Zebra finches are sometimes reared by their biological parents, but they can also parasitize the nests of a different society finch species procuring "foster parents" for their chicks. Using DNA sequencing, researchers compared the gut microbiota of chicks reared by these two types of parents along with hand-reared chicks. They found that chicks' gut microbiota mirrored those of the parents that reared them. The parents' oral cavities had the greatest contribution to the earliest gut microbes of the chicks, while in later stages, parental gut microbiota dominated in the chicks, suggesting that oral feeding is most impactful early on. These results highlight the importance of indirect maternal transmission for providing chicks with probiotic microbes and may also explain why certain species that can transfer probiotics to chicks may become preferred hosts for parasitic birds. 